\title{
Affective Meaning of Pronouns in a Russian Sample*
}

\author{
Leonid Dorfman \\ Perm State Institute of Culture, Perm, Russia
}

\begin{abstract}
The present study examines affective meaning of pronouns (in Russian) represented by the semantic differential. Of direct relevance to the present study is the theory of affective meaning propounded by Osgood. Two hypotheses were tested. According to a "magnitude" hypothesis, affective dimensions (each of evaluation, potency, and activity taken separately) differ in their magnitude across pronouns $I, M y, M e$, and They. A "structural" hypothesis stated that the affective dimensions yield latent factors across (the generality) and within (the concept-scale interaction) the pronoun concepts. Repeated-measures analysis of variance (1-way ANOVA) and confirmatory factor analysis were employed to process data. It was found that each of the evaluation and potency measures yield a significant magnitude change across the pronouns, but there was indicated no significant change across the pronouns with respect to the activity dimension. Therewith, the pronoun $M y$ gained a salient value and the pronoun They the smallest value. Using confirmatory factor analysis five models were tested. Among them one model was good fit to the data. It engaged a four-factor solution resulted in that four pronouns are latent affective distinct but related factors and the evaluation, potency, and activity are their indicators.
\end{abstract}

Keywords: personal pronouns, affective meaning, semantic differential, generality, concept-scale interaction

\section{Introduction}

Osgood, Suci, and Tannenbaum (1957) showed that an affective space is a structuring principle of the language. In the processing of language the affective dynamics has of special interest (Miron, 1969; Miron \& Osgood, 1966; Schröder, 2011). Pronouns as a grammatical form have become a popular arena of investigation related to language comprehension (e.g., Brunyé, Ditman, Giles, Holmes, \& Taylor, 2016), interpretation of pronouns (e.g., Badecker \& Straub, 2002), morphological information (e.g., van Gompel \& Liversedge, 2003), pronoun resolution (e.g., Love \& McKoon, 2011), and the like. However, affective meaning of the pronouns has been poor under examination.

The present study is aimed at assessing the affective meaning of pronouns (in Russian) represented by the semantic differential.

\section{Affective Meaning and the Semantic Differential}

Like an affective meaning is a part of the total meaning (Osgood, 1969) we may argue that an affective meaning of pronouns is a part of their total meaning. Of direct relevance to the present study is the theory of affective meaning propounded by Osgood and his coworkers (Osgood, 1969, 1980; Osgood, Suci, \& Tannenbaum, 1957). Osgood revealed the affective meaning as a relational concept, or the meaning of

\footnotetext{
* Acknowledgements: The author would like to thank Alexey Kalugin, Nils Torvald Oesterboe, and also anonymous reviewers for their constructive, valuable and helpful comments on an earlier draft of the manuscript.

Leonid Dorfman, Ph.D., D.Sc., Professor, Perm State Institute of Culture.
} 
"meaning". Its nature is multidimensional. Osgood was inspired by earlier research on synesthesia, indicated that stimuli from different modalities may have shared meanings. Based on these findings, Osgood put forward notions of imagery and metaphor, representing affective relations and associated meaning to language. Primarily, affective meaning, in this view, literally forces the metaphorical usage of words and the judgments must be metaphorically determined.

The semantic differential technique is one of the most widely used instruments for the assessment of affective meaning. It was developed based on the semantic differential paradigm. Osgood (Osgood, Suci, \& Tannenbaum, 1957) specified three "primary" dimensions of affective meaning, namely, Evaluation (E), Potency (A), and Activity (A) as shown by the factor analysis of metaphorically constructed scales. Later on, Osgood (1966) labeled them Pleasant/Unpleasant (E), Controlled/Uncontrolled (P), and Activated/Unactivated (A). As for the properly meaning, Bynner and Coxhead (1979) proposed to distinguish the "meaning" of the concepts from the "meaning" of scales. The latter is employed to indicate the former.

\section{Generality of E-P-A Versus Concept Selection}

To put the problem of our study, let's start with the fact that there have been at least three views and supporting findings on to how the affective meaning measured by the semantic differential technique can relate to concepts. From a number of problems, we draw attention to the three which collide and challenge each of them.

The long-standing and ongoing problem runs from Osgood (Osgood, 1969; Osgood, May, \& Miron, 1975; Osgood, Suci, \& Tannenbaum, 1957). He highlights the universality of the E-P-A components of meaning because they concern with most concepts. Kuusinen (1969) holds this view as well-established underlying the generality and invariance of the affective meaning structure through ratings of diverse objects. Miron (1969) gives rise to the assumption that affective $\mathrm{E}-\mathrm{P}-\mathrm{A}$ dimensions of meaning are universal and parsimonious representing common shared features across a number of concepts. Osgood, May, and Miron (1975) contend that the $\mathrm{E}-\mathrm{P}-\mathrm{A}$ structure of the affective meaning is universal and can be found in virtually any language. Recently, similar results were presented by Heise (2010) and Schröder (2011). However, the generality and invariance of the affective meaning structure occurs not to any ratings of diverse concepts and this is an issue to be still investigated and comprehended.

The second problem arises as a controversy with respect to the universality of the affective meaning dimensions independent on concept judged. This problem is labeled the "concept-scale interaction" (Heise, 1969; Kubiniec \& Farr, 1971; Mann, Phillips, \& Thompson, 1979; Osgood, Suci, \& Tannenbaum, 1957). Osgood, Suci, and Tannenbaum (1957) emphasize that the meanings of scales and their relations to other scales vary considerably with the concept being judged. Actually, the nuances of changes in meaning of the scales have been noted (e.g., Tanaka \& Osgood, 1965; Tanaka, Oyama, \& Osgood, 1963; Komorita \& Bass, 1967). Changes result in yielding quite different affective E-P-A dimensions for different concepts (Bynner \& Coxhead, 1979; Heise, 1969; Mann, Phillips, \& Thompson, 1979). Osgood (Osgood, Suci, \& Tannenbaum, 1957) suggested a general principle according to which emotionally loaded concepts being judged can arise the meaning of all scales shifting toward the E dimension. Consistent with this principle, Kubiniec and Farr (1971) found clustering of particular P and A scales with E scales when self-concepts were judged. Mann, Phillips, and Thompson (1979) came to conclusion that $\mathrm{A}$ and $\mathrm{P}$ dimensions are more sensitive to the Concept $\times$ Scale interaction than an dimension regarding to 24 concepts being rated. The concept-scale interaction has been demonstrated by studies in some specific concept areas (such as personality concepts, colors or works of art), where the scale factors 
which emerge do not follow the common three-dimensional pattern of E-P-A proposed by Osgood (Osgood, Suci, \& Tannenbaum, 1957), but instead form either some other type of affective meaning dimensions or collapse into one single E dimension (Miron \& Osgood, 1966; Komorita \& Bass, 1967). Evidence for the subject-scale interaction is established at least with respect to concepts being also rated by quite different subject groups; the meaning of the descriptive scales is different, for instance, for subjects belonging to different language/culture groups (Tanaka \& Osgood, 1965). Heise (1969) emphasizes that concept-scale interaction can arise due to the fact that a scale has different degrees of relevance for different concepts. Concept-scale interaction can also arise due to semantic shifts across concepts. Hence, semantic factor structure has to be seen a function of the stimulus concepts. Some research acknowledges the concept-scale interaction as errors of measurement (Osgood, May, \& Miron, 1975) or an artifact to be minimized (Mann, Phillips, \& Thompson, 1979), or even undermining the tradition of semantic differential research at all (for review, see Heise, 1969). Nevertheless, other researchers focus on the concept-scale interaction purposely taking into account the role of concept selection (see above). As Nordenstreng (1970) argues, when research focuses on the common characteristics of a meaning system, the concept-scale interaction effects have typically been discarded. But the more specific components of affective meaning are lost with this approach. Overall, the notion of the Concept $\times$ Scale interaction effect to some extent would seem ambiguous.

The third problem arises when a change (or stability) in meaning derived from the concept-scale interaction effect is considered as its special form. Shaw (1955) found that certain concepts seem to evoke an evaluative reaction more than others. These changes seem to have of importance for treating them as a function of the particular concept being judged. Mann, Phillips, and Thompson (1979) focus on the extent to which a given scale undergoes changes in meaning from one concept to another. They proposed the ANOVA model to be applied separately for $\mathrm{E}, \mathrm{P}$, and A scales to measure respective changes and their magnitude relying on concepts. In particular, it was found that the magnitude of the Concept $\times$ Scale effect occurs to a greater extent with $\mathrm{P}$ and A scales than with E scales. The number of studies within this line is very few and they are problematic to some extent. The fact is that they can shed light on affective meaning along its separate dimensions. Therewith, each of them is considered within or across a domain of concepts for their comparison and establishing differences. However, it is still questionable whether this approach complements or, conversely, does not match with the semantic structure based on E-P-A dimensions. The problem is not only in that the meanings of scales vary considerably with the concept being judged (Osgood, Suci, \& Tannenbaum, 1957) but also in change of each dimension and its magnitude. It would seem, though, examination of the factor structure of $\mathrm{E}-\mathrm{P}-\mathrm{A}$ dimensions and comparison of separate dimensions across concepts can be reconciled. Piotrowski (1983) reported variability in factor structure of $\mathrm{E}-\mathrm{P}-\mathrm{A}$ dimensions when the same data were analyzed across several concepts but regarding to $\mathrm{P}$ and $\mathrm{A}$ dimensions rather than to the $\mathrm{E}$ dimension. Thus, a separate analysis of each $\mathrm{E}-\mathrm{P}-\mathrm{A}$ dimension can open room to a greater comprehension of both the structure and change of affective meaning across or within the domain of concepts.

\section{Pronouns}

There is some diversity in pronoun use. In English, pronouns are morphologically marked for case (he vs. him), animacy (he vs. it), gender (he vs. she), and number (he vs. they) (van Gompel \& Liversedge, 2003). But the first-person singular pronoun has no gender, no cases, and no plural. Its forms include $I$, me, and mine (Stern, 1985). In particular, the $I$ designates the subjective aspect and the me the objective aspect of the self 
(Thass-Thienemann, 1973). Similarly, Carey et al. (2015) have emphasized use of first-person singular pronoun as subjective ( $I$ ), objective (me), and possessive (mine). McGregor (2010) highlights use of first-person singular pronoun as $I, m e, m y$. Brunyé et al. (2016) contend, in contrast, the third-person pronouns (he, she or they) link to perspective-taking which enables evaluating a situation, state, or object as it is seen by other people. For example, readers during reading may describe protagonists using the third-person pronouns (the perspective-taking) but not the first-person singular pronoun.

As was noted above, there is extensive evidence to indicate that the $\mathrm{E}-\mathrm{P}-\mathrm{A}$ dimensions can be viewed a function of the particular class of concepts employed. Among them, some grammatical forms have been specified. Osgood (1969) observed the diversity of verbs combined with nouns of various types in English. Livant (1963) demonstrated systematic differences in semantic differential ratings of the same word as a noun or a verb. In Schröder's (2011) study, subjects rated nouns, verbs, adjectives, combinations of nouns and adjectives along the $\mathrm{E}-\mathrm{P}-\mathrm{A}$ dimensions.

The list of feasible concepts encompasses also the pronouns. Kuusinen (1969) undertook in a wide range the study of ego concepts in combination with some pronouns such as myself, myself as a friend, myself as a father (or mother), myself as a child, my ideal self. The E-P-A dimensions of person concepts (along with some pronouns) were also examined, such as $m y$ best friend, a person whom $I$ very much dislike, $m y$ foreman, $m y$ colleague. The E-P-A dimensions of kinship concepts (along with some pronouns) were also investigated. Among them $m y$ father, $m y$ mother, my aunt, $m y$ uncle, my brother, $m y$ sister were engaged. Likewise, in Kubiniec and Farr's (1971) study, subjects rated their self-concept as $m y$ past, $m y$ future, $m y$ real self, and $m y$ ideal self. Similarly, in Mayerberg and Bean's (1978) study, the self-concept domain consisted of concepts including $m y$ past, $m y$ real self, and $m y$ ideal self. Davies and Stacey (1972) specified stimuli of actual self ("the kind of person $I$ actually am") and ideal self ("the kind of person $I$ would like to be"). Nordenstreng (1970) paid attention to the myself concept.

Only in some works the pronouns were employed in, so to speak, pure forms. For example, the pronoun $m e$ as a concept was used by Osgood, Suci, and Tannenbaum (1957) and the pronouns $m e$ and $I$ by Shepard (1979). However, concepts to be judged were usually taken optionally. Fortunately, some study concerned the pronouns properly. Dorfman and Goldberg (2000a) asked participants to rate each of four pronouns, namely, $I$, My, Me, and They, by means of the Osgood's semantic differential (Osgood, 1969; Osgood, Suci, \& Tannenbaum, 1957) consisting of 25 bipolar scales. Data obtained were twofold. First, the same scales were employed to judge different pronouns, for instance, "like" referred to $I, M y$, and $M e$. Other scales, second, characterized the only pronoun, for example, They was judged as "joyful" and "active". Thus, there was evidence that affective meaning can either overlap or distinct pronouns marked above.

To our knowledge, systematic studies of the E-P-A dimensions of the pronouns were disregarded. Thus, it is unclear about how the basic dimensions of affective meaning can underlie just the pronoun domain.

\section{The Present Study}

The aim of this study was to examine the personal pronouns regarding to their affective meanings represented by the E-P-A dimensions. The pronoun selection was guided by the theory of plural self (Dorfman, 2004; Dorfman \& Ogorodnikova, 2007). The plural self appears as a multifaceted mode of self-conception and entails four subselves with respective pronouns as their supposed markers. The pronoun $I$ is mostly a marker of the authorial subself (agency), the pronoun $M y$ of the embodied subself (possession), the 
pronoun "Me" of the related subself (a self-in-relation), and the pronoun They of the perspective-taking subself (taking a view seen by other people) (Dorfman \& Goldberg, 2000b). Thus, the pronouns $I, M y, M e$, and They were engaged in the present study.

Because the concept-scale interaction and change (or stability) in meaning along separate $\mathrm{E}-\mathrm{P}-\mathrm{A}$ dimensions of the marked pronouns has not yet been systematically studied, we investigated whether the pronouns (in Russian) differ in their affective meaning, when each dimension is taken separately. Because the generality of $\mathrm{E}-\mathrm{P}-\mathrm{A}$ dimensions across and within the pronoun domain has not yet been systematically tested, we also attempted to clarify the latent factor structure of the $\mathrm{E}-\mathrm{P}-\mathrm{A}$ dimensions independent (across) and dependent (within) on the pronoun concepts.

Two kinds of hypotheses were developed. The first is a "magnitude" hypothesis: Affective dimensions (the E, $\mathrm{P}$, and A taken separately) differ in their magnitude across pronouns $I, M y, M e$, and They. The second is a "structural" hypothesis with respect to the $\mathrm{E}-\mathrm{P}-\mathrm{A}$ dimensions considered as latent structures. They were divided into two facets: (A) The $\mathrm{E}-\mathrm{P}-\mathrm{A}$ latent structure appears independent on (across) the pronoun concepts; (B) The E-P-A latent structure appears dependent on (within) the pronoun concepts.

\section{Method}

\section{Participants}

Raw data were gathered from a Russian sample consisting of 145 volunteer participants recruited from Perm city universities. Because of missing data and lack of participation in different aspects of the study, 11 participants (7.58\%) were removed from subsequent analyses. The current results are based on raw data from 134 participants ( 72 women and 62 men). Their age ranged from 17 to $26, \mathrm{M}=18.70, \mathrm{SD}=1.30$. The participants received no reward or compensation for taking part in the experiment.

\section{Materials and Procedure}

Personal pronoun stimuli were administered to the participants in a random order in a number of group sessions. The participants were asked to judge meanings of each pronoun using the semantic differential.

\section{The Pronouns}

Each participant was randomly given 4 pronouns $(I, M y, M e$, and They) as stimuli and asked to rate each in terms of the semantic differential's scales (see below).

\section{The Semantic Differential}

The semantic differential technique was used to explore the affective meaning that pronouns have for the individuals being questioned. Participants were asked to give adjectival word associations as responses to a set of pronouns (I, My, Me, and They). The meaning was represented by three dimensions, namely, E, P, and A (Osgood, 1969; Osgood, Suci, \& Tannenbaum, 1957). Bipolar scales of adjectives marked by Rosnow (2000) are parsimonious and were used to tap into these dimensions. To measure the $\mathrm{E}$ dimension, scales were indicated by a set of six bipolar adjectives (bad-good, unpleasant-pleasant, negative-positive, ugly-beautiful, cruel-kind, and worthless-valuable), the P dimension by five bipolar adjective scales (weak-strong, light-heavy, small-large, soft-hard, and thin-heavy), and the A dimension by three bipolar adjective scales (slow-fast, passive-active, and dull-sharp).

Participants were asked to judge each pronoun on a seven-point scale $(3,2,1,0,-1,-2,-3)$ with reference to pairs of meanings with opposite adjectives. Responses were transformed in a range of 1-7 scores. For 
example, a magnitude " 1 " refers to a negative E, weak $P$, and slow A, and a magnitude "7" refers to a positive $\mathrm{E}$, strong $\mathrm{P}$, and fast $\mathrm{A}$. The individual ratings of the scales were averaged assigned to each dimension separately.

\section{Data Analysis}

Values of each variable were converted into z-scores. Extreme values on each variable (beyond $\mathrm{X} \pm 2 \mathrm{SD}$ ) were excluded; missing data were replaced with values known as Winsorized scores. Outlying values were reassigned to the nearest value that lies within the valid (nonoutlying) distribution (Erceg-Hurn \& Mirosevich, 2008). After this procedure was yielded, each variable had normal distribution (Kolmogorov-Smirnov test, D-max statistics).

Several statistical procedures were performed using SPSS Statistics 21 and SPSS Amos software for analyses. Descriptive statistics of the E, P, and A variables were computed. Also a $t$ test for independent samples was performed to address gender differences. Similar to Mann, Phillips, and Thompson (1979) to some extent, repeated-measures analysis of variance (1-way ANOVA) was run to examine whether there are changes in the E, P, and A variables (taken separately) of marked pronouns. F (planned comparison), Post-Hoc (Scheffe) test, and effect sizes were computed. The magnitude of effect sizes (partial eta squared values, $\eta p 2$ ) was assessed this way: .01—small effect, .06-medium effect, .14-large effect (Cohen, 1988).

Confirmatory factor analysis was employed for testing the latent factor structures of the $\mathrm{E}, \mathrm{P}$, and A variables, either independent or dependent on the pronouns. A matrix $(4 \times 3)$ was based on individual averaged ratings of the scales assigned to each of the $\mathrm{E}, \mathrm{P}$, and $\mathrm{A}$ variables separately. Five models were specified for testing. The model 1 (the E, P, and A are distinct and not related) was based on a three-factor solution. Therewith, the $\mathrm{E}, \mathrm{P}$, and $\mathrm{A}$ with four indicators each (respective to the four pronouns) were separate and not correlating latent factors across the pronouns. To provide a comparison, the model 2 (the E, P, and A are distinct and related) was based on a three-factor solution with the $\mathrm{E}, \mathrm{P}$, and $\mathrm{A}$ with four indicators each (respective to the four pronouns) as separate and correlating latent factors across the pronouns. The model 3 (a general factor model for the $\mathrm{E}-\mathrm{P}-\mathrm{A}$ ) was grounded on a one-factor solution underlying the $\mathrm{E}, \mathrm{P}$, and $\mathrm{A}$ as its shared 12 indicators (respective to the four pronouns, $3 \times 4$ ). The model 4 (the pronouns are distinct and not related) proposed a four-factor solution. Four pronouns were taken latent distinct and not correlating factors; the E, P, and A were presented as their three indicators. To provide a comparison, the model 5 (the pronouns are distinct and related) proposed a four-factor solution. Again, four pronouns were taken latent distinct but correlating factors; the E, P, and A were presented as their three indicators. There is a reason to put in the marked models the E, P, and A so way that they are latent factors themselves (independent on the pronoun concepts) or indicators of pronoun's affective meaning latent factors (dependent on the pronoun concepts). Actually, the E, P, and A taken as latent factors themselves and the $\mathrm{E}, \mathrm{P}$, and $\mathrm{A}$ taken as indicators of shared latent factors are justified as a kind of operationalization aimed at revealing difference between the $\mathrm{E}-\mathrm{P}-\mathrm{A}$ structure across and the $\mathrm{E}-\mathrm{P}-\mathrm{A}$ structure within the pronoun concepts.

To estimate model fit five indices were used to indicate model fit to the data: Steiger and Lind's root mean square error of approximation (RMSEA), the goodness-of-fit index (GFI), the adjusted goodness-of-fit index ( $A G F I$ ), the chi-square model test $\left(\chi^{2}\right)$, and the chi-square/df ratio $\left(\chi^{2} / d f\right)$ (e.g., Schermelleh-Engel, Moosbrugger, $\&$ Múller, 2003). The chi-square test is generally not suggested to evaluate model fit because of its sensitivity to non-normality, large correlations between variables, large sample sizes, and variables with high proportions of 
unique variance (Kline, 2011). Therefore, we employed RMSEA as the alternative fit index, but did not use the $\chi 2$ as a primary measure of model fit. Smaller values of $\chi^{2}, \chi^{2} / d f(<2)$, and $R M S E A$ (best $<.06$, acceptable $<.10$ ) show preferred fit (Hu \& Bentler, 1999). Values of .90 or greater have been recommended for the $G F I$ and indicate well fitting models. Values of 90 or greater also acceptable for the $A G F I$ and indicate well fitting models (Tabachnick \& Fidell, 2007). Change in model fit between models was tested by using the chi-square difference test $(\Delta \chi 2)$. The lower the value of the chi-square indicates the better the fit (Bentler, 1990). Models were analyzed using maximum-likelihood estimation which assumes multivariate normality.

\section{Results}

\section{Descriptive Statistics}

The average scores on the three measures of pronoun's E- $-\mathrm{P}-\mathrm{A}$ dimensions are shown in Table 1. As can be seen, the E, $\mathrm{P}$, and A relying on any pronoun were rated above the average point. The scores evidenced that the $\mathrm{E}$ tends to the positive end, the $\mathrm{P}$ to be strong, and A rather fast than slow. Judging the pronoun " $M e$ " women scored significantly higher than men on the $\mathrm{E}$ and $\mathrm{A}, p<.05$ but not on the $\mathrm{P}, p>.05$. Likewise, judging the pronoun "They" women scored significantly higher than men on the $\mathrm{E}$ and $\mathrm{A}, p<.01$ but not on the $\mathrm{P}, p>.05$. No significant difference was found between women and men while they judged the pronouns " $P$ " and " $M y$ " on marked measures.

Table 1

Means and Standard Deviations of Pronoun's $E-P-A$ Scores and t-Tests Between the Scores of the Participants, Women and Men

\begin{tabular}{|c|c|c|c|c|c|c|c|}
\hline \multirow{2}{*}{ Pronoun's E-P-A scores } & \multicolumn{2}{|c|}{ Total sample } & \multicolumn{2}{|c|}{ Women } & \multicolumn{2}{|c|}{ Men } & \multirow[t]{2}{*}{$t$-test } \\
\hline & $\bar{M}$ & SD & $M$ & SD & $M$ & $\mathrm{SD}$ & \\
\hline $\begin{array}{l}\text { The pronoun "I" } \\
\text { Evaluation }\end{array}$ & 5.75 & .73 & 5.80 & .72 & 5.68 & .74 & .96 \\
\hline Potency & 4.69 & .84 & 4.68 & .73 & 4.69 & .95 & .23 \\
\hline Activity & 5.34 & 1.06 & 5.32 & 1.08 & 5.36 & 1.04 & .23 \\
\hline $\begin{array}{l}\text { The pronoun "My" } \\
\text { Evaluation }\end{array}$ & 6.10 & .68 & 6.19 & .61 & 5.98 & .73 & 1.75 \\
\hline Potency & 5.13 & 1.01 & 5.18 & .92 & 5.08 & 1.11 & .56 \\
\hline Activity & 5.55 & 1.11 & 5.70 & 1.00 & 5.36 & 1.19 & 1.81 \\
\hline $\begin{array}{l}\text { The pronoun "Me" } \\
\text { Evaluation }\end{array}$ & 5.59 & .96 & 5.76 & .91 & 5.40 & .98 & $2.16^{*}$ \\
\hline Potency & 4.77 & .98 & 4.90 & .91 & 4.61 & 1.02 & 1.67 \\
\hline Activity & 5.20 & 1.18 & 5.39 & 1.10 & 4.97 & 1.22 & $2.10^{*}$ \\
\hline $\begin{array}{l}\text { The pronoun "They" } \\
\text { Evaluation }\end{array}$ & 5.27 & 1.11 & 5.50 & 1.04 & 4.99 & 1.13 & $2.73 * *$ \\
\hline Potency & 4.59 & 1.05 & 4.71 & 1.04 & 4.45 & 1.94 & 1.38 \\
\hline Activity & 5.24 & 1.12 & 5.47 & .97 & 4.97 & 1.22 & $2.62 * *$ \\
\hline
\end{tabular}

Notes: $\mathrm{M}=$ mean, $\mathrm{SD}=$ standard deviation, ${ }^{*} p<.05,{ }^{* *} p<.01$.

Although scores on several of the pronoun's E-P-A dimensions differed among women and men, the correlations for them were very similar in all cases, so only data obtained for the entire sample are shown below.

\section{Judging E, P, and A of Pronouns: A "Magnitude" Change}

Using repeated-measures ANOVAs for the E, $\mathrm{P}$, and A variables across pronouns (I, My, Me, and They), significant effects were found for two variables, namely, the $\mathrm{E}, F(1,133)=42.58, p<.001$, and $\mathrm{P}, F(1,133)=$ 
$4.43, p<.01$. For $\mathrm{E}$, the effect size was large, $\eta \mathrm{p} 2=.20$ and for $\mathrm{P}$ medium, $\eta \mathrm{p} 2=.08$. Repeated-measures ANOVA did not reveal any significant effects of the A across pronouns, $F(1,133)=2.35, p>.05$, with a small effect size, $\eta p 2=.03$.

With respect to Post-Hoc (Scheffe) test, the E obtained the greater score for the pronoun $M y$ and the least score for the pronoun They, $p<.001$. At that, there was no significant difference between the pronouns $I$ and $M e$ on the line of E scores, $p>.05$. Likewise, the $\mathrm{P}$ obtained the greater score for the pronoun $M y$ and the least score for the pronoun They, $p<.001$. Again, there was no significant difference between the pronouns $I$ and $M e$ on the line of P scores, $p>.05$.

Thus, the $\mathrm{E}$ and $\mathrm{P}$ scores yielded a significant magnitude change across the pronouns, but there was indicated no significant change across the pronouns with respect to the A dimension. Therewith, the pronoun My gained a salient value and the pronoun They the smallest value.

\section{The Model Fit and the Model Fit Comparisons}

The model fit of the models are presented in Table 2.

Table 2

Confirmatory Factor Analysis Fit Indices for the Five Models Tested the Latent Factor Structure of the $E-P-A$ Dimensions

\begin{tabular}{lllllllll}
\hline Models & RMSEA & GFI & AGFI & $\chi^{2}$ & $\chi^{2} / d f$ & $d f$ & Model fit comparison & $\Delta \chi^{2}$ \\
\hline$M_{1}$ & .14 & .80 & .67 & $161.01^{*}$ & 3.43 & 47 & - & \\
$M_{2}$ & .12 & .87 & .71 & $103.67^{*}$ & 2.96 & 35 & $X^{2}{ }_{2}{ }_{v s} X^{2}{ }_{1}$ & $57.34^{* *}$ \\
$M_{3}$ & .09 & .89 & .79 & $88.51^{*}$ & 2.11 & 42 & $X^{2}{ }_{3}{ }_{v s} X^{2}{ }_{2}$ & $15.16^{*}$ \\
$M_{4}$ & .11 & .86 & .73 & $112.43^{*}$ & 2.74 & 41 & $X^{2}{ }_{4} v s X^{2}$ & $23.92^{* *}$ \\
$M_{5}$ & .001 & .96 & .92 & $35.88^{*}$ & .90 & 40 & $X^{2}{ }_{5}{ }_{v s} X^{2}{ }_{3}$ & $52.63^{* *}$ \\
& & & & & & & $X^{2}{ }_{5} \mathrm{vs} X^{2}{ }_{4}$ & $76.55^{* *}$ \\
\hline
\end{tabular}

Notes. $M_{1}=$ the model 1 (a three-factor solution, the E, P, and A are separate and not correlating latent factors across the pronouns); $M_{2}=$ the model 2 (a three-factor solution, the $\mathrm{E}, \mathrm{P}$, and $\mathrm{A}$ are separate but correlating latent factors across the pronouns); $M_{3}=$ the model 3 (a one-factor solution across the pronouns with the $\mathrm{E}, \mathrm{P}$, and $\mathrm{A}$ as its indicators); $M_{4}=$ the model 4 (a four-factor solution; four pronouns are latent separate and not correlating factors; the E, $\mathrm{P}$, and A are their indicators); $M_{5}=$ the model 5 (a four-factor solution; four pronouns are latent separate but correlating factors; the E, P, and A are their indicators); RMSEA $=$ Steiger and Lind's root mean square error of approximation, GFI $=$ goodness-of-fit index, AGFI $=$ adjusted goodness-of-fit index, $\chi^{2}=$ chi-square statistic, $\chi^{2} / \mathrm{df}=$ chi-square $/ \mathrm{df}$ ratio, $\mathrm{df}=$ degrees of freedom, $\Delta \chi^{2}=$ chi-square difference test; $* p<.05, * * p<.001$.

The model 1 (a three-factor solution, the E, $\mathrm{P}$, and A are separate and not correlating latent factors across the pronouns), $\chi^{2}(47)=161.01, p<.001, R M S E A=.14, G F I=.80, A G F I=.67, \chi^{2} / d f=3.43$, demonstrated inadequate fit. The model 2 (a three-factor solution, the $\mathrm{E}, \mathrm{P}$, and $\mathrm{A}$ are separate but correlating latent factors across the pronouns), $\chi^{2}(35)=103.67, p<.001, R M S E A=.12, G F I=.87, A G F I=.71, \chi^{2} / d f=2.96$, still demonstrated inadequate fit. For the model 3 (a one-factor solution across the pronouns with the $\mathrm{E}, \mathrm{P}$, and $\mathrm{A}$ as its indicators), $\chi^{2}(42)=88.51, p<.001, R M S E A=.09, G F I=.89, A G F I=.79, \chi^{2} / d f=2.11$, all fit indices indicated that a one-factor model was a poor fit to the data, suggesting that the $\mathrm{E}, \mathrm{P}$, and A indicators were not directly subsumed under a one-factor across the pronouns. The assumption of the model 4 (a four-factor solution; four pronouns are latent separate and not correlating factors; the $\mathrm{E}, \mathrm{P}$, and $\mathrm{A}$ are their indicators) resulted in rather poor model fit, $\chi^{2}(41)=112.43, p<.001, R M S E A=.11, G F I=.86, A G F I=.73, \chi^{2} / d f=2.74$. The model 5 (a four-factor solution; four pronouns are latent separate but correlating factors; the E, P, and A 
are their indicators) showed the good fit, $\chi^{2}(40)=35.88, p<.001, R M S E A=.001, G F I=.96, A G F I=.92$, $\chi^{2} / d f=.90$.

Model fit comparisons resulted in the model 2 fitted the data significantly better than the model $1\left(\Delta \chi^{2}(12)\right.$ $=57.34, p<.001)$. The model 3 fitted the data significantly better than the models $2\left(\Delta \chi^{2}(7)=15.16, p<.05\right)$ and $4\left(\Delta \chi^{2}(1)=23.92, p<.001\right)$. The model 5 fitted the data significantly better than the models $3\left(\Delta \chi^{2}(2)=\right.$ $52.63, p<.001)$ and $4\left(\Delta \chi^{2}(1)=76.55, p<.001\right)$.

Overall, results support the model 5 with a four-factor solution. According to this model, four pronouns are latent distinct but related factors and the $\mathrm{E}, \mathrm{P}$, and $\mathrm{A}$ are presented as their indicators. This is the preferred model to characterize the $\mathrm{E}-\mathrm{P}-\mathrm{A}$ structure of each marked pronoun.

\section{Inspection of the Parameter Estimates for the Model 5}

Inspection of the parameter estimates for the model 5 showed $\mathrm{E}, \mathrm{P}$, and $\mathrm{A}$ strong indicators of the latent factor $I$. The parameter estimates ranged from $.45, p<.01$ to $1.21, p<.01$. Likewise, E, P, and A were indicators of the latent factor $M y$. The parameter estimates ranged from .60, $p<.001$ to $1.01, p<.001$. E, $\mathrm{P}$, and A were either indicators of the latent factor $M e$ with the parameter estimates ranged from $.56, \mathrm{p}<.001$ to 1.31 , $\mathrm{p}<.001$. The range of the parameter estimates was from .65, $p<.001$ to $1.12, p<.001$ for $\mathrm{E}$, $\mathrm{P}$, and $\mathrm{A}$ indicators of the latent factor They. Latent factors of pronouns' affective meaning correlated positively, $p$ $<.001$. Thus, results support the four-factor correlated structure underlying affective meanings of each pronoun. The model 5 is seen in Figure 1.

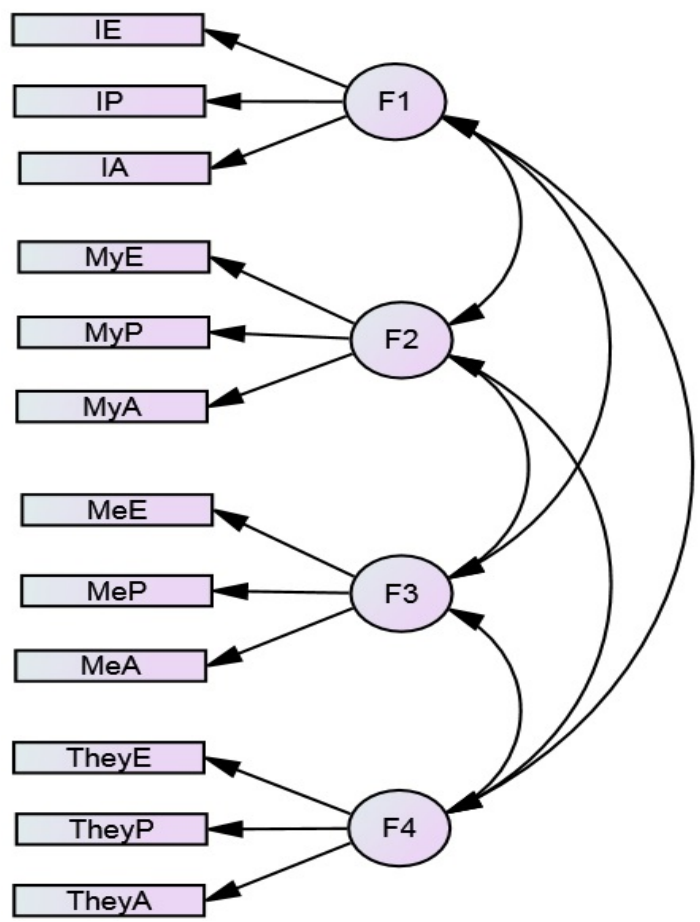

Figure 1. Confirmatory factor analysis model for the four-factor solution of pronouns' affective meaning with their E, $\mathrm{P}$, and $\mathrm{A}$ indicators.

Notes: $\mathrm{F} 1=$ the latent affective factor of the pronoun $I, \mathrm{~F} 2=$ the latent affective factor of the pronoun $M y, \mathrm{~F} 3=$ the latent affective factor of the pronoun $\mathrm{Me}, \mathrm{F} 4$ = the latent affective factor of the pronoun They. IE, IP, and IA = E, P, and A indicators of F1, MyE, $\mathrm{MyP}$, and $\mathrm{MyA}=\mathrm{E}, \mathrm{P}$, and $\mathrm{A}$ indicators of $\mathrm{F} 2, \mathrm{MeE}, \mathrm{MeP}$, and $\mathrm{MeA}=\mathrm{E}, \mathrm{P}$, and A indicators of F3, TheyE, TheyP, and TheyA = E, $\mathrm{P}$, and A indicators of F4. The latent factors positively correlate. The parameter estimates and residual coefficients are not displayed. 


\section{Discussion}

The primary aim of this study was to examine the personal pronouns $I, M y, M e$, and They regarding to their affective meaning represented by the $\mathrm{E}-\mathrm{P}-\mathrm{A}$ dimensions. This aim was twofold. First, we investigated whether the pronouns (in Russian) differ in their affective meaning when each dimension is taken separately. Second, we attempted to clarify the latent factor structure of the E-P-A dimensions independent (across) and dependent (within) on the pronoun concepts.

We found that each of the $\mathrm{E}$ and $\mathrm{P}$ measures yielded a significant magnitude change across the pronouns, but there was indicated no significant change across the pronouns with respect to the A dimension. Therewith, the pronoun $M y$ gained a salient value and the pronoun They the smallest value.

Another finding has been the extent to which a configuration of latent factor structure of the $\mathrm{E}-\mathrm{P}-\mathrm{A}$ dimensions is in effect tailoring to pronouns. In assessing the latent factor structure of the $\mathrm{E}-\mathrm{P}-\mathrm{A}$ dimensions, the model 5 demonstrated a four-factor solution, according to which four pronouns are latent affective distinct but related factors and the E, P, and A are their indicators. This model showing good fit to the data should be considered in contrast to other models with poor fits to the data. Among them were a three-factor solution, the E, P, and A are separate and not correlating latent factors across the pronouns (the model 1), a three-factor solution, the E, P, and A are separate but correlating latent factors across the pronouns (the model 2), a one-factor solution across the pronouns with the E, P, and A as its indicators (the model 3), and a four-factor solution; four pronouns are latent separate and not correlating factors; the E, P, and A are their indicators (the model 4). Inspection of the parameter estimates for the model 5 showed E, P, and A strong indicators of the latent affective pronoun factors $I, M y, M e$, and They. Therewith, the latent pronoun factors significantly and positively correlated.

The present findings pose several challenges. Established data question whether the generality of the E-P-A structure (e.g., Osgood, 1969; Osgood, May, \& Miron, 1975; Osgood, Suci, \& Tannenbaum, 1957) is the case, underlies ratings of diverse pronouns and independent on them. Actually, the respective models 1, 2 and 3, which we tested, were poor fits to the data across the pronoun domain. Statistically, hence, the $\mathrm{E}-\mathrm{P}-\mathrm{A}$ structure can scarcely be seen as highly feasible and acknowledged with respect to its universality and independence on marked pronouns. From another side, the "concept-scale interaction" effect (Heise, 1969; Kubiniec \& Farr, 1971; Mann, Phillips, \& Thompson, 1979; Osgood, Suci, \& Tannenbaum, 1957) calls also into question regarding to our data. The model 5 evidences that the $\mathrm{E}-\mathrm{P}-\mathrm{A}$ structure is sensitive to each pronoun separately but changes in the $\mathrm{E}-\mathrm{P}-\mathrm{A}$ structure did not arise across pronouns and different $\mathrm{E}-\mathrm{P}-\mathrm{A}$ structures for different pronouns did not appear. Thus, our data are scarcely consistent with something like the concept-scale interaction.

Our results, then, should be clarified in another framework bypassing either the generality of the E-P-A structure across the pronouns (the $\mathrm{E}-\mathrm{P}-\mathrm{A}$ structure is independent on pronouns) or the concept-scale interaction within pronouns (the $\mathrm{E}-\mathrm{P}-\mathrm{A}$ structure is dependent on a pronoun). Regarding to the model 5 which was good fit to our data, we propose a closer look at the changes in the $\mathrm{E}$ and $\mathrm{P}$ dimensions across the pronouns as a key to comprehend a core of the $\mathrm{E}-\mathrm{P}-\mathrm{A}$ latent factor structures of pronouns. We believe that this approach would be fruitful yielding something else than mere trivialities.

Let highlight two facets. First is sensitivity of the $\mathrm{E}-\mathrm{P}-\mathrm{A}$ structure to its separate dimensions to change. According to our data, each of the $\mathrm{E}$ and $\mathrm{P}$ (but not the A) dimension yields a significant change across the 
pronouns. This may be a case for the $\mathrm{E}-\mathrm{P}-\mathrm{A}$ is invariant in its structure despite of each of the $\mathrm{E}$ and $\mathrm{P}$ magnitude changes across the pronouns. Then, we are dealing with something like levels of the $\mathrm{E}-\mathrm{P}-\mathrm{A}$ structure on a line of magnitudes of its separate dimensions which in turn rely on a pronoun. Notice, this is not the generality phenomenon because the $\mathrm{E}-\mathrm{P}-\mathrm{A}$ structure is tied to each pronoun, not across pronouns. This is also not the concept-scale interaction because levels of the $\mathrm{E}-\mathrm{P}-\mathrm{A}$ structure cannot be reduced to its composition. Second is affective coherence of pronouns indicated by the $\mathrm{E}-\mathrm{P}-\mathrm{A}$ structure. We draw attention to the fact that latent factors of various pronouns (their $\mathrm{E}-\mathrm{P}-\mathrm{A}$ structures) are positively correlated. This entails, in particular, a suggestion that there is a common factor outside affective meaning of the pronouns.

It is worth noting that instead of to use reflexive (or intensive) pronouns (which end in -self or -selves and refer back to the subject forms of personal pronouns such as myself, themselves, etc.) we are in search for the self as a part of personality in psychology. It is not surprising because a growing body of research suggests that language can be understood as the linguistic representation of the personality concepts (e.g., Carey et al., 2015; Fitzsimons \& Kay, 2004; MA \& LIU, 2016; Makarova, 2012; McGregor, 2010; Sučylaite, 2012; XU, SUI, \& AN, 2014). The language makes available for the description of personality features. With respect to personal pronouns, they are either an important aspect for the understanding of language use or the functioning of the personality and self as its core. With few exceptions, though, little attention has been paid to the links between pronouns and the self (e.g., Adams, Bruckmüller, \& Decker, 2012; Meissner, 2008). We assume that a proximate ground of pronouns studied can stand in the plural (multifaceted) self (Dorfman, 2004; Dorfman \& Ogorodnikova, 2007). Apparently, a psychological distance between the self and pronouns can determine a magnitude of their E, $\mathrm{P}$, and A meaning change.

The plural view of the self originates from James's (1890/1902) distinction between the terms $I, M e$, and Mine. James claimed that the same brain may subserve many conscious selves, either alternate or coexisting. The $I$ - or the self-as-knower-organizes experience in a "sense of personal identity" and a "sense of a sameness" through time. In defining the $M e$ - or self-as-known — James revealed that Me confounds with the Mine. The Me or Mine are all that the person can call his or her own. These primary constituents indicate a basic structure of the self. Besides, Hermans (1996); Hermans, Kempen, and Van Loon (1992) suggested the narrative metaphor, in which story and storytelling are leading principles for the self. They applied to the Russian literary scholar Mikhail Bakhtin (1929/1973) to provide the translation of the I-Me distinction into a narrative framework based on the polyphonic view. Bakhtin showed that Dostoyevsky created the polyphonic novel. It is composed of independent and mutually opposing voices and viewpoints of characters involved in dialogue. The metaphor of the polyphonic novel entails the narrative conception of the $I$ as an author and the $M e$ as an observed actor. Thus, a multiplicity of worlds arises for one and the same individual.

To some extent, our results support viewpoints developed by James (1890/1902) and Hermans (1996); Hermans, Kempen, and Van Loon (1992). However, there are also some discrepancies in our findings compared with their suggestions. We propose that the pronoun $M y$ (possession) would be seen closer to the self than the pronoun They (perspective taking seen by other people). Therefore, My gains a salient value and They the smallest value. $I$ and $M e$ are suggested to hold an intermediate magnitude and no significant difference are revealed between them. These findings are comparable with the previous results established for separate scales of the E, P, and A dimensions (Dorfman \& Goldberg, 2000a; Dorfman \& Goldberg, 2000b). 


\section{Conclusion}

The current study was intended to investigate whether the pronouns (in Russian) differ in their affective meaning when each dimension is taken separately. It was also attempted to clarify the latent factor structure of the $\mathrm{E}-\mathrm{P}-\mathrm{A}$ dimensions independent (across) and dependent (within) on the pronoun concepts.

It was found that each of the $\mathrm{E}$ and $\mathrm{P}$ (but not the A) measure yielded a significant magnitude change across the pronouns. Therewith, the pronoun $M y$ gained a salient value and the pronoun They the smallest value. In assessing the latent factor structure of the $\mathrm{E}-\mathrm{P}-\mathrm{A}$ dimensions, it was indicated a four-factor solution, according to which four pronouns are latent affective distinct but related factors and the $\mathrm{E}, \mathrm{P}$, and $\mathrm{A}$ are their indicators. The data evidence that the $\mathrm{E}-\mathrm{P}-\mathrm{A}$ structure is sensitive to ratings of each pronoun. On the contrary, the $\mathrm{E}-\mathrm{P}-\mathrm{A}$ structure does not arise across the pronoun concepts.

\section{Limitations}

The findings of the present study are to some extent limited in generalizability. The study was conducted on a Russian sample, and it is open to question whether there are cross-cultural differences on our data or not. The sample was relatively homogeneous with respect to age, in that the tested participants were of university students.

In our study, the participants rated the pronouns $I, M y, M e$, and They using the semantic differential but rating of other pronouns by means of the semantic differential technique was disregarded. At best, obtained data can only serve as points of departure for further research at depth. Besides, it is of interest to study whether the pronouns $I, M y, M e$, and They can be particular markers of each subself labeled in the theory of plural self by Dorfman (Dorfman, 2004; Dorfman \& Ogorodnikova, 2007). We hope these lines of work will appreciably contribute to further research.

\section{References}

Adams, G., Bruckmüller, S., \& Decker, S. (2012). Self and agency in context: Ecologies of abundance and scarcity. International Perspectives in Psychology: Research, Practice, Consultation, 1(3), 141-153. doi:10.1037/a0029348

Badecker, W., \& Straub, K. (2002). The processing role of structural constraints on interpretation of pronouns and anaphors. Journal of Experimental Psychology: Learning, Memory, and Cognition, 28(4), 748-769. doi:10.1037/0278-7393.28.4.748

Bakhtin, M. (1973). Problems of Dostoevsky's poetics (2nd ed.). Ann Arbor, MI: Ardis. (Original work published 1929).

Bentler, P. M. (1990). Comparative fit indexes in structural models. Psychological Bulletin, 107(2), $238-246$. doi:10.1037//0033-2909.107.2.238

Brunyé, T. T., Ditman, T., Giles, G. E., Holmes, A., \& Taylor, H. A. (2016, February 18). Mentally simulating narrative perspective is not universal or necessary for language comprehension. Journal of Experimental Psychology: Learning, Memory, and Cognition. Advance online publication. Retrieved from http://dx.doi.org/10.1037/xlm0000250

Bynner, J., \& Coxhead, P. (1979). Some problems in the analysis of semantic differential data. Human Relations, 32(5), $367-385$. doi: $10.1177 / 001872677903200502$

Carey, A. L., Brucks, M. S., Küfner, A. C. P., Holtzman, N. S., große Deters, F., Back, M. D., ... Mehl, M. R. (2015). Narcissism and the use of personal pronouns revisited. Journal of Personality and Social Psychology, 109(3), e1-e15. Retrieved from http://dx.doi.org/10.1037/pspp0000029

Cohen, J. (1988). Statistical power for the social sciences. Hillsdale, NJ: Laurence Erlbaum and Associates.

Davies, J., \& Stacey, B. (1972). Teenagers and alcohol. London: HMSO.

Dorfman, L. Y. (2004). Self-concept: Differentiation and integration. In L. Dorfman (Ed.), Integral individuality, self-concept, and personality (pp. 96-123). Moscow: Smysl. (In Russian).

Dorfman, L. Y., \& Goldberg, I. M. (2000a). Semantic universals and codes of the plural self. In D. A. Leontiev (Ed.), Psychology of subjective semantics in fundamental and applied studies (pp. 9-11). Moscow: Smysl. (In Russian). 
Dorfman, L. Y., \& Goldberg, I. M. (2000b). Discrimination of subselves on semantic estimations of pronouns. In D. A. Leontiev (Ed.), Psychology of subjective semantics in fundamental and applied studies (pp. 40-45). Moscow: Smysl. (In Russian).

Dorfman, L., \& Ogorodnikova, A. (2007). Plural self, plural achievement motives, and creative thinking. In C. M. Dorfman and V. Petrov (Eds.), Aesthetics and innovation (pp. 125-160). Cambridge: Cambridge Scholars Press.

Erceg-Hurn, D. M., \& Mirosevich, V. M. (2008). Modern robust statistical methods: An easy way to maximize the accuracy and power of your research. American Psychologist, 63(7), 591-601. doi:10.1037/0003-066X.63.7.591

Fitzsimons, G. M., \& Kay, A. C. (2004). Language and interpersonal cognition: Causal effects of variations in pronoun usage on perceptions of closeness. Personality and Social Psychology Bulletin, 30(5), 547-557. doi:10.1177/0146167203262852

Heise, D. R. (1969). Some methodological issues in semantic differential research. Psychological Bulletin, 7, 406-422. Retrieved from http://dx.doi.org/10.1037/h0028448

Heise, D. R. (2010). Surveying cultures. Discovering shared conceptions and sentiments. Hoboken, NJ: Wiley.

Hermans, H. J. M. (1996). Voicing the self: From information processing to dialogical interchange. Psychological Bulletin, 119(1), 31-50. Retrieved from http://dx.doi.org/10.1037/0033-2909.119.1.31

Hermans, H. J. M., Kempen, H. J. G., \& Van Loon, R. J. P. (1992). The dialogical self: Beyond individualism and rationalism. American Psychologist, 47, 23-33. Retrieved from http://dx.doi.org/10.1037/0003-066X.47.1.23

Hu, L. T., \& Bentler, P. M. (1999). Cutoff criteria for fit indexes in covariance structure analysis: Conventional criteria versus new alternatives. Structural Equation Modeling, 6, 1-55. Retrieved from http://dx.doi.org/10.1080/10705519909540118

James, W. (1981). The principles of psychology (Vol. 2). Cambridge: Harvard University Press. (Original work published 1890).

Kline, R. B. (2011). Principles and practice of structural equation modeling (3rd ed.). New York, NY: Guilford Press.

Komorita, S. S., \& Bass, A. R. (1967). Attitude differentiation and evaluative scales of the semantic differential. Journal of Personality and Social Psychology, 6, 241-244. Retrieved from http://dx.doi.org/10.1037/h0024656

Kubiniec, C. M., \& Farr, S. D. (1971). Concept-scale and concept-component in interaction in the semantic differential. Psychological Reports, 28, 531-541. doi:10.2466/pr0.1971.28.2.531

Kuusinen, J. (1969). Affective and denotative structures of personality ratings. Journal of Personality and Social Psychology, 12 , 181-188. Retrieved from http://dx.doi.org/10.1037/h0027713

Livant, W. P. (1963). A comparison of noun and verb forms on the semantic differential. Journal of Verbal Learning and Verbal Behavior, 1, 357-360. doi:10.1016/S0022-5371(63)80017-1

Love, J., \& McKoon, G. (2011). Rules of engagement: Incomplete and complete pronoun resolution. Journal of Experimental Psychology: Learning, Memory, and Cognition, 37(4), 874-887. doi:10.1037/a0022932

MA, W. Y., \& LIU, X. (2016). Understanding lecturers' well-being from the aspects of self-determination theory. Journal of Literature and Art Studies, 6(2), 203-208. doi:10.17265/2159-5836/2016.02.009.

Makarova, V. (2012). The linguistic means of representing women in the Doukhobor Russian psalms. Journal of Literature and Art Studies, 2(8), 814-833.

Mann, I. T., Phillips, J. L., \& Thompson, E. G. (1979). An examination of methodological issues relevant to the use and interpretation of the semantic differential. Applied Psychological Measurement, 3(2), 213-229. doi: $10.1177 / 014662167900300211$

Mayerberg, C. K., \& Bean, A. G. (1978). Two types of factors in the analysis of semantic differential attitude data. Applied Psychological Measurement, 2(4), 469-480. doi:10.1177/014662167800200401

McGregor, S. A. (2010). The analysis of personality through language: Narcissism predicts use of shame-related words in narratives (Unpublished honor's thesis, University of Michigan, Ann Arbor, MI).

Meissner, W. W. (2008). The role of language in the development of the self III: The significance of pronouns. Psychoanalytic Psychology, 25(2), 242-256. doi:10.1037/0736-9735.25.2.242

Miron, M. S. (1969). What is it that is being differentiated by the semantic differential? Journal of Personality and Social Psychology, 12, 189-193. Retrieved from http://dx.doi.org/10.1037/h0027714

Miron, M. S., \& Osgood, C. E. (1966). Language behavior: The multivariate structure of qualification. In R. B. Cattell (Ed.), Handbook of multivariate experimental psychology. Chicago: Rand McNally.

Nordenstreng, K. (1970). Changes in the meaning of semantic differential scales: Measurement of subject-scale interaction effects. Journal of Cross-Cultural Psychology, 1(3), 217-237. doi:10.1177/135910457000100302

Osgood, C. E. (1966). Dimensionality of the semantic space for communication via facial expressions. Scandinavian Journal of Psychology, 7, 1-30. 
Osgood, C. E. (1969). On the whys and wherefores of E, P, and A. Journal of Personality and Social Psychology, 12, $194-199$. Retrieved from http://dx.doi.org/10.1037/h0027715

Osgood, C. E., May, W. H., \& Miron, M. S. (1975). Cross-cultural universals of affective meaning. Urbana: University of Illinois Press.

Osgood, C. E..Suci, G. J., \& Tannenbaum, P. H. (1957). The measurement of meaning. Urbana, IL: University of Illinois Press.

Osgood, C. E. (1980). A history of psychology in autobiography (Vol. VII, pp. 335-393). G. Lindzey (Ed.). San Francisco, CA, US: W H Freeman \& Co. Retrieved from http://dx.doi.org/10.1037/11346-009

Piotrowski, C. (1983). Factor structure on the semantic differential as a function of method of analysis. Educational and Psychological Measurement, 43(1), 183-288. doi:10.1177/001316448304300138

Rosnow, R. L. (2000). Semantic differential. Encyclopedia of Psychology, 7, 224-225. Retrieved from http://dx.doi.org/10.1037/10522-099

Schermelleh-Engel, K., Moosbrugger, H., \& Müller, H. (2003). Evaluating the fit of structural equation models: Tests of significance and descriptive goodness-of-fit measures. Methods of Psychological Research, 8, 23-74.

Schröder, T. (2011). A model of language-based impression formation and attribution among Germans. Journal of Language and Social Psychology, 30(1), 82-102. doi:10.1177/0261927X10387103

Shaw, D. R. (1955). Variation in inter-scale correlation on the semantic differential as a function of the concept judged (MS Thesis, University of Illinois at Urbana).

Shepard, L. (1979). Self-acceptance: The evaluative component of the self-concept construct. American Educational Research Journal, 16(2), 139-160. doi:10.3102/00028312016002139

Stern, D. N. (1985). The interpersonal world of the infant: A view from psychoanalysis and developmental psychology. New York: Basic Books.

Sučylaite, J. (2012). Transformative learning in language arts as a method of guidance and counseling. Journal of Literature and Art Studies, 2(7), 740-750.

Tabachnick, B. G., \& Fidell, L. S. (2007). Using multivariate statistics (5th ed.). New York: Allyn and Bacon.

Tanaka, Y., \& Osgood, C. E. (1965). Cross-culture, cross-concept and cross-subject generality of affective meaning systems. Journal of Personality and Social Psychology, 2, 143-153. Retrieved from http://dx.doi.org/10.1037/h0022392

Tanaka, Y., Oyama, T., \& Osgood, C. E. (1963). A cross-culture and cross-concept study of the generality of semantic space. Journal of Verbal Learning and Verbal Behavior, 2, 392-405. doi:10.1016/S0022-5371(63)80040-7

Thass-Thienemann, T. (1973). The interpretation of language (2 Vols.). New York: Jason Aronson.

Van Gompel, R. P. G., \& Liversedge, S. P. (2003). The influence of morphological information on cataphoric pronoun assignment. Journal of Experimental Psychology: Learning, Memory, and Cognition, 29(1), 128-139. doi:10.1037/0278-7393.29.1.128

XU, M. Y., SUI, X. D., \& AN, X. H. (2014). The fragmentation of the female selfhood in The Flight From the Enchanter. Journal of Literature and Art Studies, 4(5), 325-337. 